\title{
Haematopoietic stem cells and early lymphoid progenitors occupy distinct bone marrow niches
}

\author{
Lei Ding ${ }^{1}$ and Sean J. Morrison ${ }^{1,2}$ \\ ${ }^{1}$ Howard Hughes Medical Institute, Children's Research Institute, Department of Pediatrics, \\ University of Texas Southwestern Medical Center, Dallas, TX, 75390, USA
}

\begin{abstract}
While haematopoietic stem cells (HSCs) are commonly assumed to reside within a specialized microenvironment, or niche ${ }^{1}$, most published experimental manipulations of the HSC niche have also impacted the function of diverse restricted progenitors. This raises the fundamental question of whether HSCs ${ }^{1}$ and restricted progenitors ${ }^{2,3}$ reside within distinct, specialized niches or whether they share a common niche. Here we assess the physiological sources of the chemokine, CXCL12, for HSC and restricted progenitor maintenance. Cxcl12 ${ }^{\text {DsRed }}$ knock-in mice showed that Cxcl12 was primarily expressed by perivascular stromal cells and at lower levels by endothelial cells, osteoblasts, and some haematopoietic cells. Conditional deletion of $\mathrm{Cxcl12}$ from haematopoietic cells or Nestin-cre-expressing cells had little or no effect on HSCs or restricted progenitors. Deletion of $\mathrm{Cxcl} 12$ from endothelial cells depleted HSCs but not myeloerythroid or lymphoid progenitors. Deletion of $\mathrm{Cxcl12}$ from perivascular stromal cells depleted HSCs and certain restricted progenitors and mobilized these cells into circulation. Deletion of $\mathrm{Cxcl} 2 \mathrm{2}$ from osteoblasts depleted certain early lymphoid progenitors, but not HSCs or myeloerythroid progenitors and did not mobilize these cells into circulation. Different stem/progenitor cells thus occupy distinct cellular niches in bone marrow: HSCs in a perivascular niche and early lymphoid progenitors in an endosteal niche.
\end{abstract}

Using SLAM family markers that isolate quiescent $\mathrm{HSCs}^{4-8}$ we found that most HSCs localize adjacent to sinusoidal blood vessels in the bone marrow ${ }^{4,9,10}$. Using independent approaches others obtained similar results ${ }^{11-13}$. We therefore hypothesized that there is a perivascular niche for HSC maintenance ${ }^{4}$. Consistent with this, Stem Cell Factor (SCF) is primarily or exclusively expressed in the bone marrow by endothelial cells and perivascular stromal cells ${ }^{10}$. Conditional deletion of $S c f$ from endothelial cells or Leptin receptor (Lepr)expressing perivascular stromal cells depleted $\mathrm{HSCs}^{10}$. Combined deletion of $S c f$ from both

\footnotetext{
Users may view, print, copy, download and text and data- mine the content in such documents, for the purposes of academic research, subject always to the full Conditions of use: http://www.nature.com/authors/editorial_policies/license.html\#terms

${ }^{2}$ Correspondence: Children's Research Institute, UT Southwestern Medical Center, 5323 Harry Hines Blvd., Dallas, Texas, 75390-8502; Sean.Morrison@UTSouthwestern.edu.

AUTHOR CONTRIBUTIONS

L.D. performed all of the experiments. L.D. and S.J.M. conceived the project, designed the experiments, interpreted the results, and wrote the manuscript.
}

AUTHOR INFORMATION

The authors declare no competing financial interests. 
endothelial cells and perivascular stromal cells caused severe HSC depletion and anemia. In contrast, conditional deletion of $S c f$ from osteoblasts or haematopoietic cells did not affect HSC frequency or function. This proves there is a perivascular niche for HSC maintenance and raises the question of whether other haematopoietic progenitors reside in distinct niches.

CXCL12 is a chemokine required for HSC maintenance and retention in the bone marrow $^{11,14-17}$. Global conditional deletion of $\mathrm{Cxcl12}$, or the gene that encodes its receptor, Cxcr4, depletes HSCs from adult bone marrow ${ }^{11,14}$. CXCL12 also promotes the proliferation and maintenance of B lineage progenitors ${ }^{15,18}$ and common lymphoid progenitors (CLPs) ${ }^{19}$. CXCL12 is expressed by perivascular stromal cells, endothelial cells and osteoblasts ${ }^{11,20,21}$. Some have proposed that the physiological source of CXCL12 for HSC maintenance is osteoblasts ${ }^{14}$ while others have proposed perivascular stromal cells ${ }^{11}$; however, Cxcl12 has not yet been conditionally deleted from any candidate niche cell. Thus, the cellular sources of CXCL12 for the maintenance of HSCs and lymphoid progenitors remain uncertain.

To systematically examine the $C x c l 12$ expression pattern we generated $C x c l 12^{D s R e d}$ knockin mice by recombining DsRed-Express2 (DsRed) into the endogenous Cxcl12 locus (Supplementary Fig. 1a-c). Cxcl12 was primarily expressed by cells surrounding sinusoids throughout the bone marrow, irrespective of proximity to the endosteum (Fig. 1a-c; Supplementary Fig. 1d). Cxcl12-DsRed expression overlapped with endothelial marker staining, suggesting that endothelial cells were one source of CXCL12 (Fig. 1a-c); however, perivascular stromal cells also appeared to produce CXCL12 (Fig. 1a-c).

The perivascular $C x c l 12$ expression pattern was very similar to the $S c f$ expression pattern ${ }^{10}$. In $S c f^{g f p /+} ; C x c l 12^{D s R e d /+}$ mice we found a strong overlap in Cxcll2-DsRed and Scf-GFP expression by perivascular cells throughout the bone marrow (Fig. 1d-f). By flow cytometry, virtually all $S c f$-GFP ${ }^{+}$stromal cells were positive for Cxcl12-DsRed (Fig. 1j) and virtually all $\mathrm{Cxcl12}$-DsRed ${ }^{+}$stromal cells were positive for $S c f$-GFP (Fig. 1k). Perivascular HSC niche cells therefore produce both SCF and CXCL12.

To quantitate $\mathrm{Cxcl12}$ expression in perivascular stromal cells we sorted CD45/ Ter119-PDGFRa ${ }^{+}$mesenchymal stem/stromal cells from enzymatically dissociated bone marrow. The Lepr-Cre-expressing perivascular stromal cells that contribute to the HSC niche by secreting SCF are uniformly positive for PDGFRa ${ }^{10}$ and virtually all CD45/ Ter119-PDGFRa ${ }^{+}$bone marrow cells express Scf-GFP (Supplementary Fig. 1e, f). Approximately $90 \%$ of CD45/Ter119- ${ }^{-} \mathrm{CGFRa}^{+}$perivascular stromal cells expressed Cxcl12-DsRed (Fig. 11). By flow cytometry, approximately $70 \%$ of VE-cadherin ${ }^{+}$ endothelial cells and $0.5 \%$ of CD45/Ter $119^{+}$haematopoietic cells in the bone marrow also expressed Cxcl12-DsRed (Fig. 1m, n and Supplementary Fig. 1g).

In contrast to $S c f$, which is not detectably expressed by osteoblasts ${ }^{10}$, we did observe very low levels of Cxcl12-DsRed expression by bone-lining cells (Fig. 1g-i). Almost 70\% of Col2.3-GFP ${ }^{+}$osteoblasts isolated from enzymatically digested bone from Col2.3-GFP; Cxcl12 $2^{\text {SRed/+ }}$ mice expressed low levels of Cxcl12-DsRed (Fig. 10). 
By quantitative reverse transcription polymerase chain reaction (qRT-PCR), EYFP ${ }^{+}$ perivascular stromal cells from Lepr-cre; loxpEYFP mice expressed Cxcl12 at $~ 15,000$-fold the level observed in unfractionated bone marrow (Fig. 1p). VE-cadherin ${ }^{+}$endothelial cells, Col2.3-GFP ${ }^{+}$osteoblasts, and $\mathrm{Cxcl12}$-DsRed ${ }^{+}$haematopoietic cells expressed significantly lower levels of $\mathrm{Cxcl12}$ at $\sim 120$ fold, $\sim 13$ fold, and $\sim 3$ fold the levels observed in bone marrow cells (Fig. 1p).

We generated a floxed allele of $\mathrm{Cxcl12}\left(\mathrm{Cxcl1} 2^{f l}\right)$ that led to a frameshift upon recombination (Supplementary Fig. 2a-c). The floxed allele itself did not appear to have any phenotype as unrecombined $\mathrm{Cxcl1} 2^{f l f f l}$ mice were born and matured into adulthood in normal numbers with normal HSC frequency and haematopoiesis (Supplementary Fig. 2df). We recombined $\mathrm{Cxcl1} 2^{f l}$ in the germline with $\mathrm{CMV}$-cre mice to generate a $\mathrm{Cxcll2^{- }}$ predicted null allele. $\mathrm{Cxcl12^{+/- }}$ mice were born in expected numbers (Supplementary Fig. $2 \mathrm{~g}$ ) with normal cellularity, B cell frequency, and HSC frequency in the bone marrow and spleen (Supplementary Fig. $2 \mathrm{~h}-\mathrm{j}$ ). In contrast, $\mathrm{Cxcll}^{-/-}$progeny were not born alive (Supplementary Fig. 2g) consistent with the known perinatal lethal phenotype of Cxcl12 deficient mice ${ }^{15}$.

Global deletion of Cxcl12 by administering tamoxifen to 8-week old adult Ubc-creER; Cxcll $2^{f l f l}$ mice significantly reduced white blood cell counts (Supplementary Fig. 4a), lymphocyte frequencies (Supplementary Fig. 4b), bone marrow cellularity (Supplementary Fig. 4c) and $\mathrm{CD} 150^{+} \mathrm{CD} 48^{-}$Lineage ${ }^{-} \mathrm{Sca}^{+}{ }^{+} \mathrm{CKit}^{+} \mathrm{HSC}^{4}$ frequency (Supplementary Fig. 4d). Bone marrow cells from Ubc-creER; Cxcll $2^{f l f l}$ mice also gave significantly lower levels of donor cell reconstitution in all major haematopoietic lineages upon transplantation into irradiated mice (Supplementary Fig. 4e). Consistent with an independently targeted $\mathrm{Cxcl1} 2^{\mathrm{fl}}$ allele $^{14}$, these results demonstrate CXCL12 promotes adult HSC maintenance and lymphopoiesis.

HSCs do not express $\mathrm{Cxcl12}$ by flow cytometry (Supplementary Fig. 4f). However, since some other haematopoietic cells expressed Cxcl12-DsRed (Fig. 1n, p), we conditionally deleted $\mathrm{Cxcl12}$ from all haematopoietic cells in Vav1-cre; Cxcl12flfl mice. Recombination was highly efficient in Vav1-cre; Cxcll $2^{f l f l}$ HSCs (Supplementary Fig. 5a). Adult Vav1-cre; Cxcll $2^{f l f l}$ mice had normal cellularity, HSC frequency (Supplementary Fig. 4g, h), lineage composition in the bone marrow and spleen (Supplementary Fig. 5b), and reconstituting potential in irradiated mice (except for a modest decline in $\mathrm{T}$ cell reconstitution; Supplementary Fig. 4i). Cells from the bone marrow, spleen and blood of Vav1-cre; $\mathrm{Cxcl1} 2^{f l f l}$ mice formed normal numbers of haematopoietic colonies in culture (Supplementary Fig. 5c). We were unable to detect HSCs in the blood of Vav1-cre; Cxcll $2^{f l f l}$ mice when we competitively transplanted 600,000 mononucleated blood cells into irradiated mice (data not shown).

Adult Vav1-cre; $C x c l 12^{f l f l}$ mice also had normal frequencies of CD150-CD48- $\mathrm{LSK}$ multipotent progenitors (MPPs; see Supplementary Figure 3 for flow cytometry gates and references for each cell population), Flt $3{ }^{+}$LSK lymphoid-primed MPPs (LMPPs), $\mathrm{CD} 34^{+} \mathrm{Fc} \gamma \mathrm{R}^{-}$Lineage $^{-} \mathrm{Sca}^{-} \mathrm{cKit}^{+}$common myeloid progenitors (CMPs), Lineage ${ }^{-} \mathrm{Sca} 1^{\text {low }}{ }_{\mathrm{CKit}}{ }^{\text {low }} \mathrm{Flt} 3^{+} \mathrm{IL} 7 \mathrm{Ra}^{+}$common lymphoid progenitors (CLPs), 
CD34- ${ }^{-} \mathrm{c} \gamma \mathrm{R}^{-}$Lineage ${ }^{-} \mathrm{Sca}^{-}{ }^{-} \mathrm{cKit}^{+}$megakaryocytic/erythroid progenitors (MEPs), and $\mathrm{CD} 34^{+} \mathrm{Fc} \gamma \mathrm{R}^{+}$Lineage ${ }^{-} \mathrm{Sca}^{-}{ }^{-} \mathrm{Kit}^{+}$granulocyte/macrophage progenitors (GMPs) (Supplementary Fig. 4j, k). Committed B lineage progenitors including $\mathrm{B} 220^{+} \mathrm{sIgM}{ }^{-} \mathrm{CD} 43^{+} \mathrm{CD} 24^{-}$pre-pro B cells, B220 ${ }^{+} \mathrm{SIgM}^{-} \mathrm{CD} 43^{+} \mathrm{CD} 24^{+}$pro B cells,

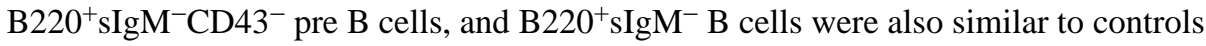
(Supplementary Fig. 41). Cxcll2 expression by haematopoietic cells is therefore not required for the maintenance or retention of HSCs or restricted progenitors in adult bone marrow.

Nestin-cre; Cxcl1 $2^{f l f l}$ mice also had normal bone marrow and spleen cellularity, HSC frequency, and haematopoietic lineage composition (Supplementary Fig 6a, b). Bone marrow cells from Nestin-cre; $\mathrm{Cxcl1} 2^{\mathrm{fl} f f l}$ mice were indistinguishable from control cells in their capacity to give long-term multilineage reconstitution of irradiated mice (Supplementary Fig. 6c). The bone marrow of Nestin-cre; Cxcll $2^{f l f l}$ mice had normal frequencies of MPPs, LMPPs, CMPs, CLPs, MEPs, GMPs, committed B lineage progenitors (Supplementary Fig. 6d-f). Nestin-cre; Cxcll $2^{f l f l}$ mice also had normal frequencies of colony-forming progenitors in the bone marrow, spleen, and blood (Supplementary Fig. 6gi). We were unable to detect HSCs in the blood of these mice in competitive reconstitution assays (data not shown). Nestin-cre expressing cells are therefore not a physiologically important source of CXCL12 for the maintenance of HSCs or restricted progenitors in the bone marrow. While HSC niche cells do not express endogenous Nestin, Nestin-cre, or Nestin-creER, they do appear to express the Nestin-GFP transgene ${ }^{10}$, consistent with previously published results ${ }^{12}$.

Given that $\mathrm{Cxcl12}$ from haematopoietic cells was not required by HSCs or restricted progenitors (Supplementary Fig. 4g-1), Tie2-cre; Cxcll $2^{f l f l}$ mice allowed us to test whether Cxcl12 from endothelial cells is physiologically important. Adult Tie2-cre; Cxcl12 $2^{f l f l}$ mice had normal bone marrow and spleen cellularity (Fig. 2a), blood cell counts, and lineage composition in the bone marrow and spleen (Supplementary Fig. 7a, b). However, the frequency of $\mathrm{CD} 150^{+} \mathrm{CD} 48^{-}$Lineage ${ }^{-} \mathrm{Sca}{ }^{+}{ }^{+} \mathrm{Cit}^{+} \mathrm{HSCs}$ in the bone marrow of Tie2-cre; $\mathrm{Cxcl1} 2^{f l f l}$ mice was significantly lower than in littermate controls (Fig. 2b). Bone marrow cells from Tie2-cre; Cxcl1 $2^{f l f l}$ mice also gave significantly lower levels of donor cell reconstitution in all major hematopoietic lineages upon transplantation into irradiated mice (Fig. 2c). Endothelial cells are therefore a physiologically important source of CXCL12 for HSC maintenance. This likely reflects a requirement for CXCL12 produced by postnatal bone marrow endothelial cells because Cxcl12 deficient mice do not exhibit HSC depletion during fetal development, only in postnatal bone marrow ${ }^{17,22}$. HSCs from Tie2-cre; Cxcl1 $2^{f l f l}$ mice had normal frequencies of $\mathrm{BrdU}^{+}$and Annexin $\mathrm{V}^{+}$cells, suggesting that HSCs may be depleted in these mice through premature differentiation (Supplementary Fig. $7 \mathrm{c}, \mathrm{d})$.

Tie2-cre; $C x c l 12^{f l f l}$ mice had normal frequencies of MPPs, LMPPs, CMPs, CLPs, MEPs, GMPs, and B lineage progenitors in the bone marrow (Fig. 2d-f) and colony-forming progenitors in the bone marrow, spleen, and blood (Fig. 2g-i). We were unable to detect HSCs in the blood of these mice in competitive reconstitution assays (data not shown). CXCL12 produced by endothelial cells is therefore not required for the retention of HSCs or most restricted progenitors in bone marrow. 
We deleted $\mathrm{Cxcl12}$ from perivascular stromal cells in the bone marrow using Lepr-cre ${ }^{10}$. Lepr-cre; Cxcll2 $2^{f l-}$ mice had normal bone marrow and spleen cellularity (Fig. 3a), normal blood cell counts, and normal lineage composition in the bone marrow and spleen (Supplementary Fig. 7e, f). CD $150^{+} \mathrm{CD} 48^{-}$Lineage $^{-} \mathrm{Sca} 1^{+} \mathrm{cKit}^{+} \mathrm{HSC}$ frequency was normal in the bone marrow of Lepr-cre; Cxcl12 $2^{f l /-}$ mice (Fig. 3b) and the reconstituting capacity of bone marrow cells in irradiated mice was normal (Fig. 3c). The bone marrow of Lepr-cre; Cxcl1 $2^{f l-}$ mice also had normal frequencies of MPPs, LMPPs, CMPs, CLPs, MEPs, GMPs, and B lineage progenitors (Fig 3d-f). However, the frequencies of HSCs and colonyforming progenitors were significantly elevated in the blood and spleen of Lepr-cre; Cxcl1 $2^{f l /}$ mice (Fig. 3b, h-j). This demonstrates that CXCL12 expression by Lepr ${ }^{+}$ perivascular cells is required to retain HSCs and colony-forming progenitors in the bone marrow.

Col2.3-Cre recombines genes in fetal and adult osteoblasts ${ }^{10,23}$. Col2.3-cre; Cxcl1 $2^{\text {flffl }}$ mice had normal bone marrow and spleen cellularity, normal $\mathrm{CD} 150^{+} \mathrm{CD} 48^{-} \mathrm{Lineage}^{-} \mathrm{Sca}{ }^{+} \mathrm{CKit}^{+}$ HSC frequency in the bone marrow and spleen (Fig. 4a, b), normal blood cell counts, normal lineage composition in the bone marrow and spleen (Supplementary Fig. 8a, b). Col2.3-cre; Cxcll $2^{f l f l}$ bone marrow cells gave normal levels of donor myeloid reconstitution, but significantly lower levels of $\mathrm{T}$ and $\mathrm{B}$ cell reconstitution in irradiated mice relative to control cells (Fig. 4c). In contrast, purified HSCs from Col2.3-cre; Cxcll $2^{f l f l}$ mice gave similar levels of reconstitution in all lineages as control HSCs (Fig. 4d and Supplementary Fig. 8c). This suggests that the reduction in $\mathrm{T}$ and $\mathrm{B}$ cell reconstitution from the bone marrow of Col2.3-cre; $\mathrm{Cxcl1} 2^{f l f l}$ mice reflected the depletion of early lymphoid progenitors.

The bone marrow of Col2.3-cre; $C x c l 12^{f l f l}$ mice had normal frequencies of MPPs, CMPs, MEPs, and GMPs (Fig. 4e) but significantly fewer CLPs and IL7Ra ${ }^{+}$LMPPs - cells thought to be specified for lymphoid differentiation ${ }^{24,25}$ (Fig. 4f, g). The thymus in Col2.3-cre; Cxcll $2^{f l f l}$ mice had a normal frequency of early thymic progenitors (B220- $\mathrm{Gr}^{-}$Ter1 $19^{-} \mathrm{CD} 4^{-} \mathrm{CD} 8^{-} \mathrm{CD} 44^{+} \mathrm{CKit}^{+} \mathrm{CD} 25^{-}$ETPs; Supplementary Fig. 8d, e) and the bone marrow had a normal frequency of committed B lineage progenitors (Fig. 4h). This suggests that homeostatic mechanisms can restore normal frequencies of downstream lymphoid progenitors despite depletion of primitive lymphoid progenitors. CLPs from Col2.3-cre; Cxcl12flffl mice had normal frequencies of $\mathrm{BrdU}^{+}$and Annexin $\mathrm{V}^{+}$cells, suggesting that these cells might be depleted by premature differentiation (Supplementary Fig. 8f, g).

Col2.3-cre; Cxcl1 $2^{f l f l}$ mice had normal frequencies of myeloerythroid colony-forming progenitors in the bone marrow, spleen, and blood (Fig. 4i) and we were unable to detect HSCs in the blood of these mice in competitive reconstitution assays (data not shown). CXCL12 from osteoblasts is thus required for the maintenance of certain early lymphoid progenitors but not for the maintenance or bone marrow retention of HSCs or myeloerythroid progenitors.

In stained bone marrow sections we found $30 \%$ of Lineage ${ }^{-} \mathrm{IL} 7 \mathrm{Ra}^{+}$cells at the endosteum, adjacent to bone-lining cells (Fig. $4 \mathrm{j}$ and Supplementary Fig. 9). Only 5\% of all bone marrow cells were adjacent to the endosteum in the sections we examined, so these early 
lymphoid progenitors were 6 times more likely than the average bone marrow cell to be adjacent to endosteum. Both functional genetic data and localization data therefore suggest there is an endosteal niche for a subset of early lymphoid progenitors.

To test whether both HSCs and early lymphoid progenitors would be depleted by deleting Cxcl12 in perivascular stromal cells and in osteoblasts we used $\operatorname{Prxl} 1-\mathrm{cre}^{26}$. Greenbaum et al. discovered that Prxl-Cre deletes broadly in perivascular stromal cells as well as in osteoblasts $^{27}$. Consistent with this, $\operatorname{Prx} 1$-Cre recombined a conditional reporter in osteoblasts as well as in $95 \pm 4 \%$ CD45/Ter119-PDGFRa ${ }^{+}$perivascular stromal cells (Supplementary Fig. 10a-c) but not in bone marrow endothelial cells (Supplementary Fig. 10d). Thus, Prxl-Cre recombined more broadly in perivascular stromal cells than Lepr-Cre, which recombined a conditional reporter in $70 \pm 9 \%$ of $\mathrm{CD} 45 / \mathrm{Ter} 119^{-} \mathrm{PDGFRa}^{+}$cells (Supplementary Fig. 1f). Consistent with our results with other Cre alleles, Prxl-cre; Cxcll $2^{f l f l}$ mice exhibited significant reductions in bone marrow cellularity (Fig. $4 \mathrm{k}$ ) and in the frequencies of HSCs (Fig. 4l), CLPs, and IL7Ra ${ }^{+}$LMPPs (Fig. 4m). The bone marrow of

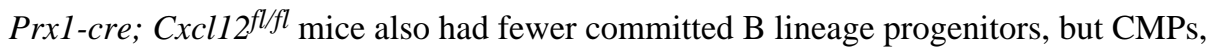
MEPs, and GMPs were normal (Supplementary Fig. 10e, f).

Our data indicate that HSCs depend upon a perivascular niche created by endothelial cells and Lepr-cre/Prxl-cre-expressing perivascular stromal cells, while some early lymphoid progenitors depend upon an endosteal niche created by osteoblasts, and committed B lineage progenitors depend upon a distinct perivascular niche created by Prxl-cre-expressing stromal cells but not endothelial cells. Greenbaum et al. ${ }^{27}$ come to similar conclusions. Osteoblasts promote the proliferation and differentiation of lymphoid progenitors in culture and conditional ablation of osteoblasts in vivo acutely depletes lymphoid progenitors but not $\mathrm{HSCs}^{28,29}$. Conditional deletion of G protein alpha from osteoblasts depletes B lineage progenitors but not other haematopoietic progenitors ${ }^{30}$. Our data are thus consistent with a number of prior studies, including our recent work ${ }^{10}$, in suggesting that osteoblasts create a niche for certain early lymphoid progenitors but not for HSCs.

\section{SUPPLEMENTARY METHODS}

\section{Mice}

Targeting vectors for making $\mathrm{Cxcl1} 2^{\mathrm{DsRed}}$ and $\mathrm{Cxcl} 12^{\mathrm{fl}}$ mice were generated by recombineering ${ }^{31}$. Linearized targeting vector was electroporated into C57BL-derived Bruce4 ES cells. Correctly targeted clones were identified by Southern blotting. Following expansion, these ES cell clones were injected into C57BL/6-Tyr ${ }^{\mathrm{c}-2 \mathrm{~J}}$ blastocysts. Chimeric mice were bred with C57BL/6-Tyr ${ }^{\mathrm{c}-2 \mathrm{~J}}$ mice to obtain germline transmission. The Frt-flanked Neo selection cassette was removed by subsequent mating with Flpe mice ${ }^{32}$. The resulting mice were backcrossed for at least three generations onto a C57BL/Ka background. Mice used in this study included Ubc-creER ${ }^{42}, C M V$-cre $e^{43}$, Vav1-cre ${ }^{44}$, Nestin-cre $^{45}$, Tie2-cre $e^{46}$, Lepr-cre ${ }^{47}$, Prxl-cre $e^{26}$, and LoxpEYFP $P^{48}$ (all from Jackson Laboratory). Scfff ${ }^{\text {gf } /+}$ mice were described previously ${ }^{10}$. Col2.3-cre mice ${ }^{23}$ were obtained from Drs. F. Liu and B. Kream (University of Connecticut). Col2.3-GFP mice ${ }^{49}$ were obtained from Dr. D. Rowe. Tamoxifen chow (Harlan) containing tamoxifen citrate (Spectrum Chemical) at 400mg/kg, 
with 5\% sucrose added, was administrated to mice for 2-4 months to induce recombination by CreER.

All mice were housed in the Unit for Laboratory Animal Medicine at the University of Michigan or in the Animal Resource Center at the University of Texas Southwestern Medical Center. All protocols were approved by the University of Michigan Committee on the Use and Care of Animals and by the UT Southwestern Institutional Animal Care and Use Committee.

\section{PCR genotyping}

Primers for genotyping of the $\mathrm{Cxcl1} 2^{\mathrm{DsRed}}$ allele were: OLD322, $5^{\prime}$ -

GATGCGCGTCAGAGACCCC-3'; OLD355, 5'-GCGCAGAGCTGCGAGCCTTTC-3'; and OLD 415, $5^{\prime}$-CTTGAAGCGCATGAAGGGCTTG- $3^{\prime}$. Primers for genotyping of the Cxcl12 $2^{\mathrm{fl}}$ allele were: OLD312, 5'-GAGCCCAGAACTCTGCCACC-3'; and OLD313, 5'TCTTGCAAAGACCATCCCCTC- $3^{\prime}$. Primers for genotyping of the $\mathrm{CxCl12^{- }}$ allele were: OLD312, 5'-GAGCCCAGAACTCTGCCACC-3', and OLD353, 5'ACTCTGAGTGAGATATTCACATC-3'.

\section{Long-term competitive reconstitution assay}

Adult mice were administered a minimum lethal dose of radiation using a Cesium 137 GammaCell40 Irradiator (MDS) or an XRAD 320 x-ray irradiator (Precision X-Ray Inc.) to deliver two doses of 540 rads (total 1080 rads) at least 2 hours apart. C57BL/6-SJL (CD45.1) mice were used as recipient mice in transplantation experiments. Cells were injected into the retro-orbital venus sinus of anaesthetized mice. Blood cells were subjected to Ficoll centrifugation prior to transplantation to isolate mononucleated cells.

Mononucleated cells were counted prior to transplantation either using a CBC machine or a hemocytometer with Turks solution. In most experiments recipient mice were maintained on antibiotic water (neomycin sulfate $1.11 \mathrm{~g} / \mathrm{l}$ and polymixin B $0.121 \mathrm{~g} / \mathrm{l}$ ) for 14 days after transplantation and then switched to regular water. Recipient mice were bled from 4 to 16 weeks after transplantation to examine the levels of donor-derived myeloid, B and T cells in their blood. Red blood cells were lysed with ammonium chloride potassium buffer before antibody staining. The antibodies used to analyze donor chimerism in the blood were antiCD45.1 (A20), anti-CD45.2 (104), anti-Gr1 (8C5), anti-Mac1 (M1/70), anti-B220 (6B2) and anti-CD3 (KT31.1).

\section{Flow cytometry}

Bone marrow cells were isolated by flushing the long bones or by crushing the long bones with mortal and pestle in $\mathrm{Ca}^{2+}$ and $\mathrm{Mg}^{2+}$ free HBSS with $2 \%$ heat-inactivated bovine serum. Spleen cells were obtained by crushing the spleen between two glass slides. The cells were dissociated to a single cell suspension by gently passing through a $25 \mathrm{G}$ needle then filtering


CD150 (TC15-12F12.2), anti-CD48 (HM48-1), anti-CD41 (MWReg30), anti-Sca1 (E13-161.7), anti-cKit (2B8) and the following antibodies against lineage markers (antiTer119, anti-B220 (6B2), anti-Gr1 (8C5), anti-CD2 (RM2-5), anti-CD3 (17A2), anti-CD5 (53-7.3) and anti-CD8 (53-6.7)). Haematopoietic progenitors were identified by flow 
cytometry using the following antibodies: anti-Sca1 (E13-161.7), anti-cKit (2B8) and the following antibodies against lineage markers (anti-Ter119, anti-B220 (6B2), anti-Gr1 (8C5), anti-CD2 (RM2-5), anti-CD3 (17A2), anti-CD5 (53-7.3) and anti-CD8 (53-6.7)), anti-CD34 (RAM34), anti-CD135 (Flt3) (A2F10), anti-CD16/32 (FcrR) (93), anti-CD127 (IL7Ra) (A7R34), anti-CD24 (M1/69), anti-CD43 (1B11), anti-B220 (6B2), anti-IgM (II/41), antiCD3 (17A2), anti-Gr1 (8C5), anti-Mac1 (M1/70), anti-CD41 (MWReg30), anti-CD71 (C2), anti-Ter119, anti-CD44 (IM7) and anti-CD25 (PC61). DAPI was used to exclude dead cells.

For flow cytometric analysis of $S c$-GFP+ or Lepr-cre; loxpEYFP+ stromal cells, bone marrow was flushed using HBSS- with $2 \%$ bovine serum. Then whole bone marrow was digested with Collagenase IV $(200 \mathrm{U} / \mathrm{ml})$ and DNase I $(200 \mathrm{U} / \mathrm{ml})$ at $37^{\circ} \mathrm{C}$ for $15 \mathrm{~min}$. Samples were then stained with antibodies and analyzed by flow cytometry. Anti-CD140a (APA5), anti-CD45 (30F-11) and anti-Ter119 antibodies were used to isolate perivascular stromal cells. For analysis of bone marrow endothelial cells, mice were i.v. injected with 10ug/mouse Alexa Fluor 647 conjugated anti-VE-cadherin antibody (BV13, eBiosciences $)^{51}$. Ten minutes later, the long bones were removed and bone marrow was flushed, digested and stained as above. Samples were run on FACSAria or FACSCanto II flow cytometers. Data were analyzed by FACSDiva (BD) or FlowJo (Tree Star) software. Osteoblasts were dissociated as described ${ }^{50}$.

\section{Methylcellulose cultures}

Cells were sorted into methylcellulose culture medium (3434, Stemcell Technology) and incubated at $37^{\circ} \mathrm{C}$ as described ${ }^{9}$. Blood cells were obtained by Ficoll centrifuge according to manufacture's recommendation before plating (GE Healthcare).

\section{Immunostaining bone sections}

Freshly dissected long bones were fixed in a Formalin-based fixative at $4^{\circ} \mathrm{C}$ for 3 hours. Then the bones were embedded in $8 \%$ gelatin in PBS. Samples were snap frozen with liquid nitrogen and stored at $-80^{\circ} \mathrm{C}$. Bones were sectioned using CryoJane (Instrumedics). Sections were dried overnight at room temperature (RT) and stored at $-80^{\circ} \mathrm{C}$. Sections were re-hydrated in PBS for 5 min before immunostaining. 5\% goat serum in PBS was used for blocking. Primary antibodies were applied to the slides for 1 hour at RT, followed by secondary antibody incubation for 30min at RT with repeated washes in between. Slides were mounted with anti-fade prolong gold (Invitrogen) and images were acquired on an Olympus IX81 microscope or a Zeiss LSM 780 confocal microscope. Primary antibodies were: chicken-anti-GFP (Aves), rat-anti-mouse pan-endothelial cell antigen (Meca32, Biolegend), rat-anti-endoglin (eBioscience), rat-anti-RFP (AlleleBiotechnology) and goatanti-osteopontin (R\&D). For staining $\mathrm{Lin}^{-} \mathrm{IL}_{\mathrm{Ra}} \mathrm{R}^{+}$cells in bone marrow sections, mice were intravenously injected with 2ug Alexa Fluor 647 conjugated monoclonal antibody against IL7Ra (A7R34, Biolegend). After 5 minutes, the mice were killed, and long bones were dissected, fixed, sectioned, and stained with antibodies against Lineage markers (CD2, CD3, CD5, CD8, Ter119, Gr1 and B220) as described above. Images were acquired using a Zeiss LSM 780 confocal microscope. 


\section{Quantitative reverse transcription PCR}

Cells were sorted directly into Trizol. Total RNA was extracted according to manufacture's instructions. Total RNA was subjected to reverse transcription using SuperScript III (Invitrogen). Quantitative real-time PCR was run using SYBR green on a LightCycler 480 (Roche). $\beta$-actin was used to normalize the RNA content of samples. Primers used in this study were: Cxcl12: OLD35, 5'-TGCATCAGTGACGGTAAACCA-3' and OLD36, 5'GTTGTTCTTCAGCCGTGCAA-3'. $\beta$-actin: OLD27, 5'-GCTCTTTTCCAGCCTTCCTT- $3^{\prime}$ and OLD28, 5'-CTTCTGCATCCTGTCAGCAA-3'.

\section{Supplementary Material}

Refer to Web version on PubMed Central for supplementary material.

\section{Acknowledgments}

This work was supported by the Howard Hughes Medical Institute (HHMI) and the National Heart, Lung and Blood Institute (5R01-HL097760). L.D. was supported by a Helen Hay Whitney Foundation Fellowship and by HHMI. We thank M. White and D. Adams for flow cytometry, E. Hughes and T. Saunders at the University of Michigan transgenic core for helping to generate $\mathrm{Cxcl1} 2^{\mathrm{DsRed}}$ and $\mathrm{Cxcl1} 2^{f l}$ mice, and S. Grove, R. Coolon, S. Manning, M. Gross and K. Correll for managing the mouse colony.

\section{References}

1. Morrison SJ, Spradling AC. Stem cells and niches: mechanisms that promote stem cell maintenance throughout life. Cell. 2008; 132:598-611. [PubMed: 18295578]

2. Akashi K, Traver D, Miyamoto T, Weissman IL. A clonogenic common myeloid progenitor that gives rise to all myeloid lineages. Nature. 2000; 404:193-197. [PubMed: 10724173]

3. Kondo M, Weissman IL, Akashi K. Identification of clonogenic common lymphoid progenitors in mouse bone marrow. Cell. 1997; 91:661-672. [PubMed: 9393859]

4. Kiel MJ, Yilmaz OH, Iwashita T, Terhorst C, Morrison SJ. SLAM Family Receptors Distinguish Hematopoietic Stem and Progenitor Cells and Reveal Endothelial Niches for Stem Cells. Cell. 2005; 121:1109-1121. [PubMed: 15989959]

5. Wilson A, et al. Hematopoietic stem cells reversibly switch from dormancy to self-renewal during homeostasis and repair. Cell. 2008; 135:1118-1129. [PubMed: 19062086]

6. Morita Y, Ema H, Nakauchi H. Heterogeneity and hierarchy within the most primitive hematopoietic stem cell compartment. J Exp Med. 2010; 207:1173-1182. [PubMed: 20421392]

7. Kiel MJ, et al. Haematopoietic stem cells do not asymmetrically segregate chromosomes or retain BrdU. Nature. 2007; 449:238-242. [PubMed: 17728714]

8. Foudi A, et al. Analysis of histone 2B-GFP retention reveals slowly cycling hematopoietic stem cells. Nature Biotechnology. 2009; 27:84-90.

9. Kiel MJ, Radice GL, Morrison SJ. Lack of evidence that hematopoietic stem cells depend on Ncadherin-mediated adhesion to osteoblasts for their maintenance. Cell Stem Cell. 2007; 1:204-217. [PubMed: 18371351]

10. Ding L, Saunders TL, Enikolopov G, Morrison SJ. Endothelial and perivascular cells maintain haematopoietic stem cells. Nature. 2012; 481:457-462. [PubMed: 22281595]

11. Sugiyama T, Kohara H, Noda M, Nagasawa T. Maintenance of the hematopoietic stem cell pool by CXCL12-CXCR4 chemokine signaling in bone marrow stromal cell niches. Immunity. 2006; 25:977-988. [PubMed: 17174120]

12. Mendez-Ferrer $S$, et al. Mesenchymal and haematopoietic stem cells form a unique bone marrow niche. Nature. 2010; 466:829-834. [PubMed: 20703299]

13. Lo Celso C, et al. Live-animal tracking of individual haematopoietic stem/progenitor cells in their niche. Nature. 2009; 457:92-96. [PubMed: 19052546] 
14. Tzeng YS, et al. Loss of Cxc112/Sdf-1 in adult mice decreases the quiescent state of hematopoietic stem/progenitor cells and alters the pattern of hematopoietic regeneration after myelosuppression. Blood. 2011; 117:429-439. [PubMed: 20833981]

15. Nagasawa T, et al. Defects of B-cell lymphopoiesis and bone-marrow myelopoiesis in mice lacking the CXC chemokine PBSF/SDF-1. Nature. 1996; 382:635-638. [PubMed: 8757135]

16. Petit I, et al. G-CSF induces stem cell mobilization by decreasing bone marrow SDF-1 and upregulating CXCR4. Nature Immunology. 2002; 3:687-694. [PubMed: 12068293]

17. Ara T, et al. Long-term hematopoietic stem cells require stromal cell-derived factor- 1 for colonizing bone marrow during ontogeny. Immunity. 2003; 19:257-267. [PubMed: 12932359]

18. Nagasawa T, Kikutani H, Kishimoto T. Molecular cloning and structure of a pre-B-cell growthstimulating factor. Proceedings of the National Academy of Sciences USA. 1994; 91:2305-2309.

19. Nie Y, Han YC, Zou YR. CXCR4 is required for the quiescence of primitive hematopoietic cells. The Journal of experimental medicine. 2008; 205:777-783. [PubMed: 18378795]

20. Ponomaryov T, et al. Induction of the chemokine stromal-derived factor-1 following DNA damage improves human stem cell function. Journal of Clinical Investigation. 2000; 106:1331-1339. [PubMed: 11104786]

21. Dar A, et al. Chemokine receptor CXCR4-dependent internalization and resecretion of functional chemokine SDF-1 by bone marrow endothelial and stromal cells. Nature Immunology. 2005; 6:1038-1046. [PubMed: 16170318]

22. Kawabata K, et al. A cell-autonomous requirement for CXCR4 in long-term lymphoid and myeloid reconstitution. Proceedings of the National Academy of Sciences USA. 1999; 96:5663-5667.

23. Liu F, et al. Expression and activity of osteoblast-targeted Cre recombinase transgenes in murine skeletal tissues. Int J Dev Biol. 2004; 48:645-653. [PubMed: 15470637]

24. Adolfsson J, et al. Identification of Flt3+ lympho-myeloid stem cells lacking erythromegakaryocytic potential a revised road map for adult blood lineage commitment. Cell. 2005; 121:295-306. [PubMed: 15851035]

25. Inlay MA, et al. Ly6d marks the earliest stage of B-cell specification and identifies the branchpoint between B-cell and T-cell development. Genes \& Development. 2009; 23:2376-2381. [PubMed: 19833765]

26. Logan M, et al. Expression of Cre Recombinase in the developing mouse limb bud driven by a Prxl enhancer. Genesis. 2002; 33:77-80. [PubMed: 12112875]

27. Greenbaum A, et al. Cxcl12 production by early mesenchymal progenitors is required for hematopoietic stem cell maintenance. Nature. 2012 In Press.

28. Visnjic D, et al. Hematopoiesis is severely altered in mice with an induced osteoblast deficiency. Blood. 2004; 103:3258-3264. [PubMed: 14726388]

29. Zhu J, et al. Osteoblasts support B lymphocyte commitment and differentiation from hematopoietic stem cells. Blood. 2007; 109:3706-3712. [PubMed: 17227831]

30. Wu JY, et al. Osteoblastic regulation of B lymphopoiesis is mediated by Gs \{alpha\}-dependent signaling pathways. Proceedings of the National Academy of Sciences USA. 2008; 105:1697616981.

\section{SUPPLEMENTARY REFERENCES}

31. Liu P, Jenkins NA, Copeland NG. A highly efficient recombineering-based method for generating conditional knockout mutations. Genome Research. 2003; 13:476-484. [PubMed: 12618378]

32. Rodriguez CI, et al. High-efficiency deleter mice show that FLPe is an alternative to Cre-loxP. Nature Genetics. 2000; 25:139-140. [PubMed: 10835623]

33. Kiel MJ, Yilmaz OH, Morrison SJ. CD150- cells are transiently reconstituting multipotent progenitors with little or no stem cell activity. Blood. 2008; 111:4413-4414. [PubMed: 18398056]

34. Karsunky H, Inlay MA, Serwold T, Bhattacharya D, Weissman IL. Flk2+ common lymphoid progenitors possess equivalent differentiation potential for the B and T lineages. Blood. 2008; 111:5562-5570. [PubMed: 18424665] 
35. Hardy RR, Carmack CE, Shinton SA, Kemp JD, Hayakawa K. Resolution and characterization of pro-B and pre-pro-B cell stages in normal mouse bone marrow. Journal of Experimental Medicine. 1991; 173:1213-1225. [PubMed: 1827140]

36. Koch U, Radtke F. Mechanisms of T cell development and transformation. Annual Review of Cell and Developmental Biology. 2011; 27:539-562.

37. Morikawa S, et al. Prospective identification, isolation, and systemic transplantation of multipotent mesenchymal stem cells in murine bone marrow. Journal of Experimental Medicine. 2009; 206:2483-2496. [PubMed: 19841085]

38. Katayama Y, et al. Signals from the sympathetic nervous system regulate hematopoietic stem cell egress from bone marrow. Cell. 2006; 124:407-421. [PubMed: 16439213]

39. Mendez-Ferrer S, Lucas D, Battista M, Frenette PS. Haematopoietic stem cell release is regulated by circadian oscillations. Nature. 2008; 452:442-447. [PubMed: 18256599]

40. Yamazaki S, et al. Nonmyelinating Schwann cells maintain hematopoietic stem cell hibernation in the bone marrow niche. Cell. 2011; 147:1146-1158. [PubMed: 22118468]

41. Tokoyoda K, Egawa T, Sugiyama T, Choi BI, Nagasawa T. Cellular niches controlling B lymphocyte behavior within bone marrow during development. Immunity. 2004; 20:707-718. [PubMed: 15189736]

42. Ruzankina Y, et al. Deletion of the developmentally essential gene ATR in adult mice leads to agerelated phenotypes and stem cell loss. Cell Stem Cell. 2007; 1:113-126. [PubMed: 18371340]

43. Schwenk F, Baron U, Rajewsky K. A cre-transgenic mouse strain for the ubiquitous deletion of loxP-flanked gene segments including deletion in germ cells. Nucleic Acids Research. 1995; 23:5080-5081. [PubMed: 8559668]

44. de Boer J, et al. Transgenic mice with hematopoietic and lymphoid specific expression of Cre. European Journal of Immunology. 2003; 33:314-325. [PubMed: 12548562]

45. Tronche F, et al. Disruption of the glucocorticoid receptor gene in the nervous system results in reduced anxiety. Nature Genetics. 1999; 23:99-103. [PubMed: 10471508]

46. Koni PA, et al. Conditional vascular cell adhesion molecule 1 deletion in mice: impaired lymphocyte migration to bone marrow. J Exp Med. 2001; 193:741-754. [PubMed: 11257140]

47. DeFalco J, et al. Virus-assisted mapping of neural inputs to a feeding center in the hypothalamus. Science. 2001; 291:2608-2613. [PubMed: 11283374]

48. Srinivas $\mathrm{S}$, et al. Cre reporter strains produced by targeted insertion of EYFP and ECFP into the ROSA26 locus. BMC Dev Biol. 2001; 1:4. [PubMed: 11299042]

49. Kalajzic Z, et al. Directing the expression of a green fluorescent protein transgene in differentiated osteoblasts: comparison between rat type I collagen and rat osteocalcin promoters. Bone. 2002; 31:654-660. [PubMed: 12531558]

50. Semerad CL, et al. G-CSF potently inhibits osteoblast activity and CXCL12 mRNA expression in the bone marrow. Blood. 2005; 106:3020-3027. [PubMed: 16037394]

51. Butler JM, et al. Endothelial cells are essential for the self-renewal and repopulation of Notchdependent hematopoietic stem cells. Cell Stem Cell. 2010; 6:251-264. [PubMed: 20207228] 

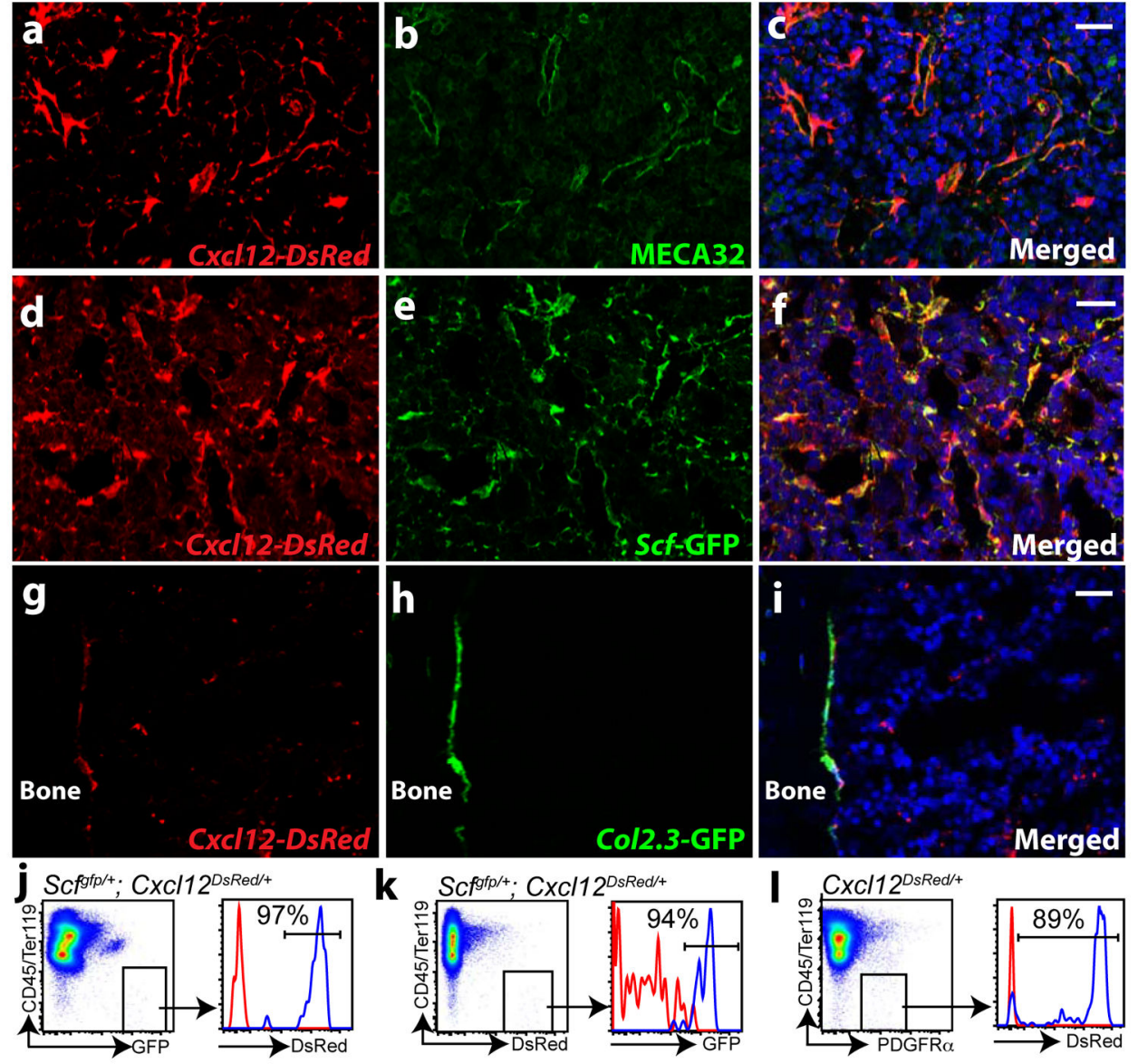

k

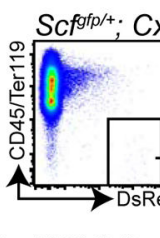

$2^{D s R e d / 4}$

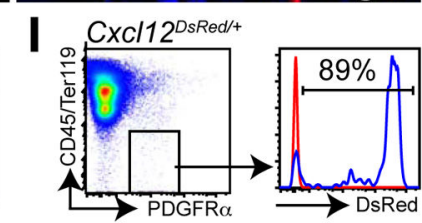

$\mathbf{m}_{\mathrm{CxCl} 12^{\text {DsRed/+ }}}$

n $\mathrm{CxCl} 12^{\mathrm{DsRed} /+}$
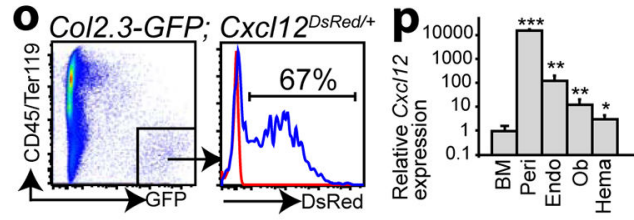

Figure 1. Endothelial cells and perivascular stromal cells are the major sources of $\mathrm{Cxcl12}$ in bone marrow

a-c, In $\mathrm{Cxcl12} 2^{\mathrm{DsRed} /+}$ mice, DsRed was primarily expressed by perivascular cells throughout the bone marrow. $\mathbf{d}-\mathbf{f}, \mathrm{Cxcl12-DsRed}$ and $S c f$-GFP expression strongly overlapped around sinusoids throughout the bone marrow of $\mathrm{Scfgfp/+} ; \mathrm{Cxcll} 2^{\mathrm{DsRed} /+}$ mice. g-i, Col2.3-GFP ${ }^{+}$bone-lining osteoblasts expressed Cxcl12-DsRed in the bone marrow of Col2.3-GFP ${ }^{+} ; \mathrm{Cxcll}_{2}{ }^{\mathrm{DsRed} /+}$ mice. Nuclei were stained with 4', 6-diamidino-2phenylindole (DAPI, blue) in $\mathbf{c}, \mathbf{f}$ and i. j, CD45/Ter119-Scf-GFP ${ }^{+}$perivascular stromal cells expressed Cxcl12-DsRed in $S c f^{g f p /+} ; \mathrm{Cxcll}^{\mathrm{DsRed} /+}$ (blue histogram) but not in $\mathrm{Scfgfp/+}$ control marrow (red histogram). k, CD45/Ter119- Cxcl12-DsRed ${ }^{\text {high }}$ cells expressed $S c f$ GFP in Scffop/+ $; C x c l 12^{D s R e d /+}$ (blue) but not in Cxcl12 $2^{\text {DsRed/+ }}$ control marrow (red). l, CD45/Ter119-PDGFRa ${ }^{+}$perivascular stromal cells expressed Cxcl12-DsRed in Cxcl12 $2^{D s R d /+}$ (blue) but not control (+/+; red) marrow. m, VE-cadherin ${ }^{+}$endothelial cells expressed Cxcl12-DsRed in Cxcl12 ${ }^{D s R e d /+}$ (blue) but not control (+/+; red) marrow. n, $0.5 \%$ of CD45/Ter1 $19^{+}$haematopoietic cells expressed Cxcl12-DsRed in Cxcl12 ${ }^{D s R e d /+}$ but not in 
control marrow (Supplementary Fig. 1g). o, CD45/Ter119- Col2.3-GFP ${ }^{+}$osteoblasts from enzymatically dissociated bone expressed Cxcl12-DsRed in Col2.3-GFP; Cxcl12DsRed/+ (blue) but not Col2.3-GFP control (red) mice. p, Cxcl12 transcript levels by qRT-PCR in different subpopulations of bone marrow cells $(\mathrm{n}=3-6)$. BM, whole bone marrow cells. Peri, $\mathrm{EYFP}^{+}$perivascular stromal cells from Lepr-cre; loxpEYFP mice. Endo, VE-cadherin ${ }^{+}$bone marrow endothelial cells. Ob, Col2.3-GFP ${ }^{+}$osteoblasts. Hema, CD45/Ter119+Cxcl12-

$\operatorname{DsRed}^{+}$haematopoietic cells. All data represent mean \pm s.d. from at least three independent experiments. Two-tailed student's t-tests were always used to assess statistical significance $(* \mathrm{P}<0.05, * * \mathrm{P}<0.01, * * * \mathrm{P}<0.001)$. Scale bars are $20 \mathrm{um}$. 

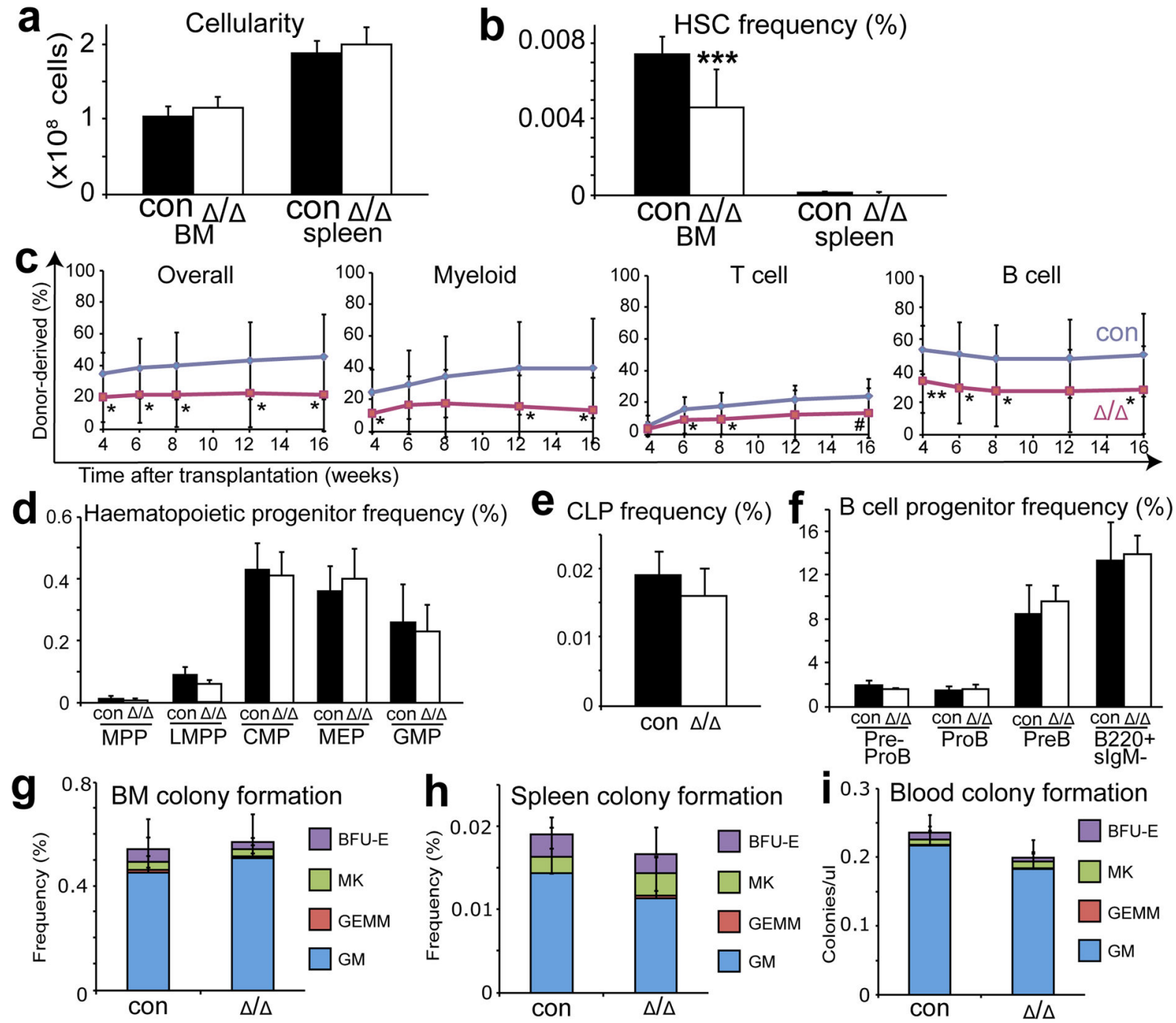

Figure 2. CXCL12 produced by endothelial cells promotes HSC maintenance

a, Bone marrow and spleen cellularity $(n=8-9)$ and (b) HSC frequency in Tie2-cre; Cxcl12 flffl mice versus littermate controls $(\mathrm{n}=10-11)$. c, $3 \times 10^{5}$ donor bone marrow cells from Tie2-cre; $\mathrm{Cxcl1} 2^{f l / f l}$ mice gave significantly lower levels of donor myeloid, B, and T cell reconstitution in irradiated mice (three experiments with a total of 12-14 recipients per genotype). d-f, Tie2-cre; Cxcl12 flffl mice had normal frequencies of MPPs, LMPPs, CMPs, MEPs, GMPs (d), CLPs (e), and committed B lineage progenitors in bone marrow (f) $(\mathrm{n}=3-$

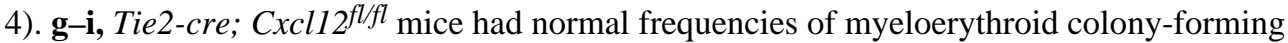
progenitors in bone marrow $(\mathbf{g})$, spleen $(\mathbf{h})$, and blood $(\mathbf{i})(\mathrm{n}=3-6) . \Delta$, recombined $C x c l 12^{f l}$ allele; con, negative control mice with $+/+$ or fl/+ or fl/fl Cxcl12 genotypes (without $\mathrm{cre}$ ). Data are mean \pm s.d. $(* \mathrm{P}<0.05, * * \mathrm{P}<0.01, * * * \mathrm{P}<0.001, \#=0.057)$. 

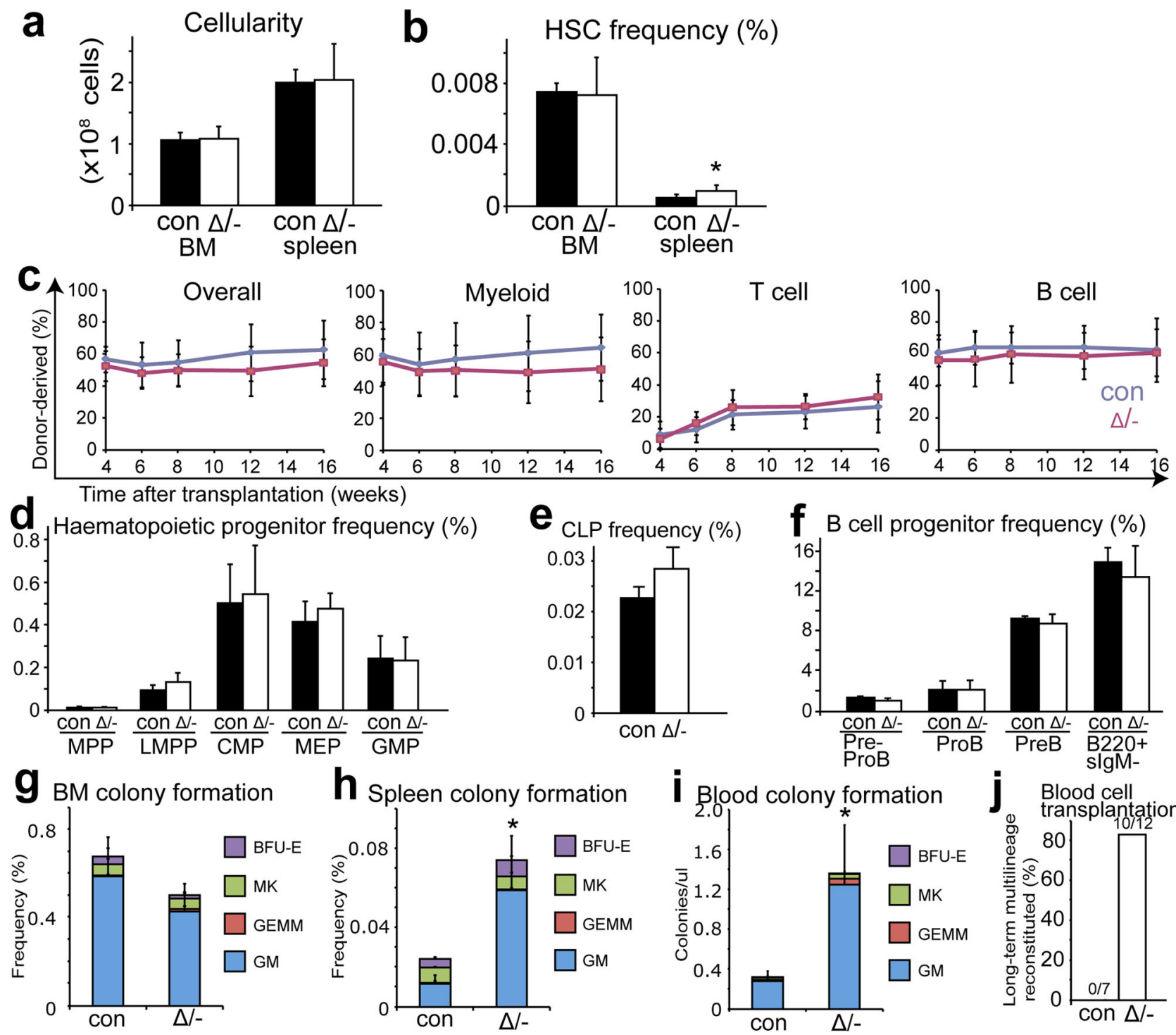

Figure 3. CXCL12 produced by Lepr-expressing perivascular stromal cells retains HSCs and colony-forming progenitors in the bone marrow

$\mathbf{a}, \mathbf{b}$, Cellularity $(\mathbf{a}, \mathrm{n}=6)$ and HSC frequency $(\mathbf{b}, \mathrm{n}=6-7)$ in the bone marrow and spleen of Lepr-cre; Cxcll2 fl/- mice and littermate controls. c, $3 \times 10^{5}$ bone marrow cells from Lepr-

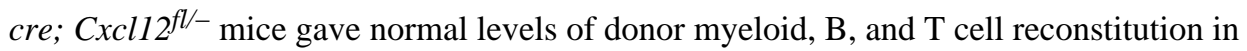
irradiated mice (three experiments with a total of 15 recipient mice per genotype). d-f, Leprcre; $\mathrm{Cxcl1} 2^{\mathrm{fl} / \mathrm{-}}$ mice had normal frequencies of MPPs, LMPPs, CMPs, MEPs, GMPs (d), CLPs (e), and committed B lineage progenitors in bone marrow (f) $(n=3)$. $\mathbf{g - i}$, Lepr-cre; $\mathrm{Cxcl1} 2^{\mathrm{fl} /-}$ mice had normal frequencies of myeloerythroid colony-forming progenitors in bone marrow (g) but significantly increased frequencies in spleen $(\mathbf{h})$, and blood (i) $(\mathrm{n}=3-5)$. j, $6 \times 10^{5}$ mononucleated blood cells from Lepr-cre; $\mathrm{Cxcll} 2^{\mathrm{fl} / \text { - }}$ mice gave long-term multilineage reconstitution in irradiated mice, while blood cells from littermate controls did not. $\Delta$, recombined $C x c l 12^{f l}$ allele; -, germline deleted $C x c l 12$ allele or $C x c l 12^{D s R e d}$ allele; con, control mice. Data represent mean \pm s.d. $(* \mathrm{P}<0.05, * * \mathrm{P}<0.01, * * * \mathrm{P}<0.001)$. 

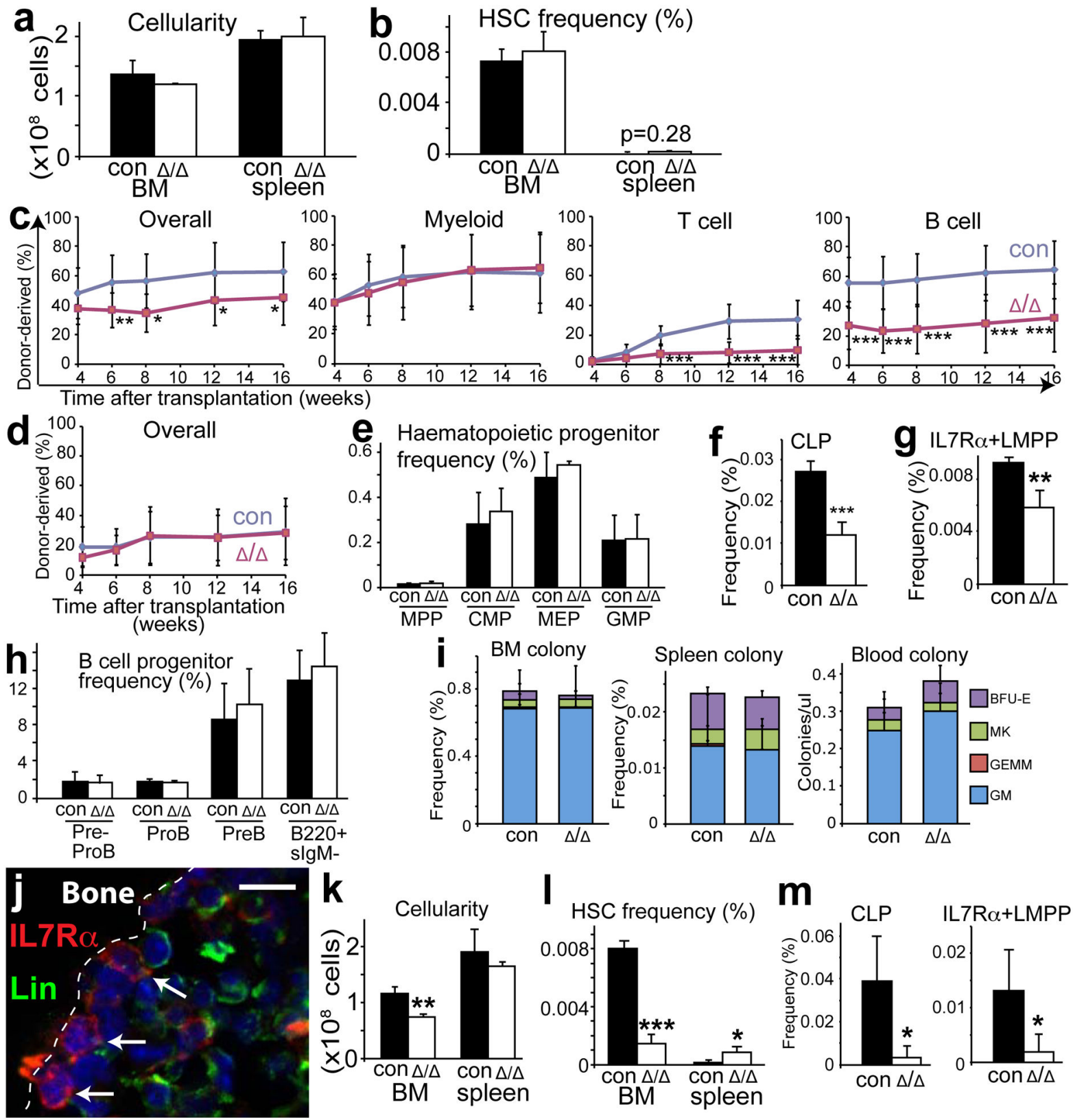

Figure 4. CXCL12 produced by osteoblasts promotes the maintenance of early lymphoid progenitors but not HSCs

$\mathbf{a}, \mathbf{b}$, Cellularity $(\mathbf{a}, \mathrm{n}=4)$ and HSC frequency $(\mathbf{b}, \mathrm{n}=4)$ in the bone marrow and spleen of Col2.3-cre; $\mathrm{Cxcl} 12^{f l f f l}$ mice and littermate controls. c, $3 \times 10^{5}$ bone marrow cells from

Col2.3-cre; Cxcl12flfl mice gave significantly lower levels of donor cell reconstitution in the

$\mathrm{T}$ and $\mathrm{B}$ cell lineages but not in the myeloid lineage relative to control bone marrow cells (three experiments with a total of 13-14 recipients per genotype). d, 20

$\mathrm{CD} 150^{+} \mathrm{CD} 48^{-}{ }^{-}$ineage ${ }^{-} \mathrm{Sca}{ }^{+}{ }^{-} \mathrm{Kit}^{+} \mathrm{HSCs}$ from Col2.3-cre; $\mathrm{Cxcl1} 2^{\text {fllfl }}$ mice gave normal donor cell reconstitution (three experiments with a total of 14-15 recipients per genotype), including normal levels of myeloid, B, and T cells (Supplementary Fig. 8c). e-h, Col2.3- 
cre; $\mathrm{Cxcl1} 2^{f l f l}$ bone marrow had normal frequencies of MPPs, CMPs, MEPs, GMPs (e), and committed B lineage progenitors (h) but significantly reduced frequencies of CLPs (f) and IL7Ra+LMPPs (g) (n=3-5). i, Col2.3-cre; Cxcll $2^{f l f f l}$ mice had normal frequencies of myeloerythroid colony-forming progenitors in the bone marrow, spleen, and blood $(n=3-6)$. j, Some $\mathrm{Lin}^{-} \mathrm{IL} 7 \mathrm{Ra}^{+}$early lymphoid progenitors were adjacent to the endosteum. $\mathbf{k}-\mathbf{m}$, Prxl-cre; $\mathrm{Cxcl1} 2^{f l f l}$ mice exhibited significant reductions in bone marrow cellularity (k, $\mathrm{n}=3-4)$ and the frequencies of HSCs (l), CLPs, and IL7Ra ${ }^{+}$MMPPs $(\mathbf{m}, \mathrm{n}=4-5) . \Delta$, recombined $C x c l 12^{f l}$ allele; con, control mice. $* \mathrm{P}<0.05, * * \mathrm{P}<0.01, * * * \mathrm{P}<0.001$. 